\title{
FINITARY IMBEDDINGS OF CERTAIN GENERALIZED SAMPLE SPACES $\left({ }^{1}\right)$
}

\author{
BY \\ MARIE A. GAUDARD AND ROBERT J. WEAVER
}

\begin{abstract}
A generalized sample space each of whose subspaces has as its logic an orthomodular poset is called an HD sample space. In this paper it is shown that any HD sample space may be imbedded in a natural way in a generalized sample space which is HD and at the same time admits a full set of dispersion free weight functions.
\end{abstract}

I. Introduction. Of fundamental importance to the mathematical formulation of quantum mechanics (see for example [7]) are abstract measures, or "states," which closely resemble the concrete probability measures on the lattice of propositions of a classical probability space. In most treatments, however, these abstract measures do not arise from a sample space of outcomes for some experimental procedure. In the recent work of D. J. Foulis, C. H. Randall, and others [2] , [3], [4], [8], [9], [10], [11], [12], [13], a "generalized sample space" represents the outcome set for a general situation involving the empirical sciences. "Generalized weight functions" on these spaces give rise to probability measures on the partially ordered set of "generalized propositions" which faithfully reflect the construction of classical probability measures and at the same time form abstract measures serving the needs of quantum mechanics.

Our discussion of generalized sample spaces begins with the notion of an orthogonality space: an orthogonality space consists of a pair $(X, \perp)$ where $X$ is a nonempty set and $\perp$ is a symmetric antireflexive binary relation on $X$ called orthogonality. If $(X, \perp)$ is an orthogonality space and $A \subset X$, we define $A^{\perp}=$ $\{x \in X \mid x \perp a$ for all $a \in A\}, A^{\perp \perp}=\left(A^{\perp}\right)^{\perp}$, etc. If $x \in X$, let $x^{\perp}$ denote $\{x\}^{\perp}$. A subset $W$ of $X$ is an orthogonal set provided distinct elements of $W$ are pairwise

Received by the editors November 11, 1973 and, in revised form, February 22, 1974. AMS (MOS) subject classifications (1970). Primary 05B99, 05C99, 60B99.

Key words and phrases. Empirical logic, generalized sample space, HD sample space, logic, complete orthomodular lattice, operation, orthogonal set, scattered set, finitary sample space, idealized point.

( $)$ The results in this paper were obtained jointly during research for the first author's Senior Honors Thesis at Mount Holyoke College under the direction of the second author. The authors express deep appreciation to Professors David J. Foulis and Charles H. Randall of the University of Massachusetts at Amherst for their useful suggestions.

The work of the second author was supported in part by a Mount Holyoke College Faculty Grant. 
orthogonal; let $O(X, 1)=\{W \subset X \mid W$ is an orthogonal set $\}$. Let $E(X, 1)=$ $\left\{E \in O(X, 1) \mid E^{\perp}=\varnothing\right\}$; that is, $E(X, 1)$ is the collection of all sets which are maximal as orthogonal sets under set inclusion. Thinking of $X$ as the outcome set for some collection of experiments, and " $\perp$ " as a sort of "operational rejection," one can consider elements of $E(X, 1)$ as sets of mutually exhaustive outcomes resulting from some experimental procedure. Hence, in keeping with frequently used terminology, we call orthogonal sets events and maximal orthogonal sets operations. A generalized sample space consists of an orthogonality space together with its collection of operations.

In the Foulis-Randall interpretation, the generalized sample space becomes the framework within which numerous related operations can be represented and their interrelationships studied. A thorough and interesting discussion of this can be found in [3], [8], and [10]. It should be mentioned that our discussion here is not as general as that which appears in [3], [10], [11], and [13], where not all maximal orthogonal subsets of $X$ are included in the collection of "admissible operations."

An immediate example of an orthogonality space is given by the collection of nonzero vectors of any inner product space under the usual definition of orthogonality. Another example is the collection of vertices of any unoriented graph, where distinct vertices are defined to be orthogonal if they are joined by an edge. Conversely, any finite orthogonality space $(X, \perp)$ can be represented by a finite graph: we let $X$ be the set of vertices, and we join two vertices by an edge whenever these vertices are orthogonal. Thus, the following graphs correspond to orthogonality spaces in the manner described:

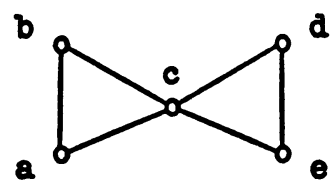

(a)

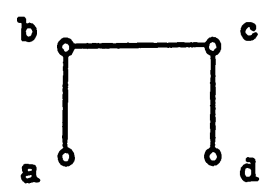

(b)

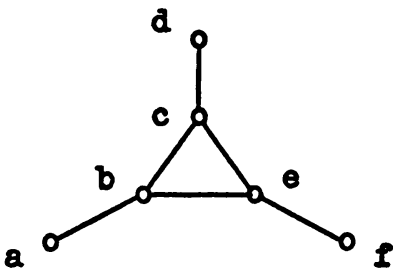

(c)

("Hook")

FIGURE 1

If $(X, \perp)$ is any orthogonality space and $D \in O(X, 1)$, one can interpret $D^{\perp}$ as the set of outcomes which refute the event $D$, and $D^{\perp \perp}$ as the set which confirm the event $D$. In this light, $D^{\Perp}$ becomes a generalized proposition asserting that the event $D$ is confirmed whenever an execution of the appropriate experiment yields a result in $D^{\Perp \perp}$. Furthermore, if $C$ and $D$ are events such that $C^{\Perp} \subset D^{\Perp}$, then 
any outcome confirming $C$ also confirms $D$, whence $C^{\perp \Perp}$ is said to imply $D^{\Perp}$. The logic of $(X, 1)$ is defined to be the collection $\Pi(X, 1)=\left\{D^{\perp \perp} \mid D \in O(X, 1)\right\}$ ordered by implication, or equivalently by set inclusion.

For any sample space $(X, \perp)$, the logic $(\Pi(X, \perp), \subset)$ clearly forms a partially ordered set. However, most formulations of quantum mechanics require that the so-called "quantum logic" be at least an orthomodular poset (for lattice theoretic terminology, see [1]), and Mackey [7] requires that it be a complete orthomodular lattice. Thus sample spaces whose logics exhibit these properties are of particular interest in applications to quantum physics. An orthogonality space whose logic is an orthomodular poset is called a Dacey space, after J. C. Dacey who first studied such spaces [2]. A Dacey space each of whose subsets is itself a Dacey space (under the inherited orthogonality) is called a hereditary Dacey space, or HD space. An HD sample space has the additional property that its logic is a complete orthomodular lattice.

In Figure 1, (a) and (c) are Dacey spaces, while (b) is not. It can be shown that an orthogonality space is an HD space if and only if it contains no four element subset isomorphic to a hook (Figure 1(b)). Let us agree to denote any hook with vertices arranged as in Figure 1(b) by the ordered quadruple $(a, b, c, d)$. Then clearly Figure 1(c), while a Dacey space, is not an HD space: it contains, for example, the hook $(d, c, e, f)$.

If $(X, \perp)$ is an orthogonality space, then we say that distinct elements $x, y$ in $X$ are scattered, or sharp, provided $x \notin y^{\perp}$. We denote this relation $x \# y$. Clearly $(X, \#)$ forms an orthogonality space, and it will be called the complement of $(X, 1)$. The sets in $O(X, \#)$ are called scattered sets, while those in $E(X, \#)$ are called maximal scattered sets. The collection of maximal scattered sets in $(X, 1)$ is denoted $S(X, 1)$.

Letting a solid line denote orthogonality between elements of $(X, \perp)$ and a broken line represent the \# relation, we immediately observe that the complement of a hook $(a, b, c, d)$ in $(X, 1)$ is the hook $(b, d, a, c)$ in $(X, \#)$ (Figure 2).

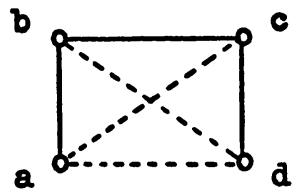

FIGURE 2

This implies the result, of fundamental importance in what follows, that an orthogonality space is an HD space if and only if its complement is an HD space. 
A weight function on $(X, 1)$ is a mapping $\omega: X \rightarrow[0,1]$ such that $\Sigma_{e \in E} \omega(e)=1$ for every $E$ in $E(X, 1)$, where the sum is taken in the unordered sense for nonnegative real numbers. If $\omega$ is a weight function on $X$, then the regular state $\alpha_{\omega}$ corresponding to $\omega$ is the mapping $\alpha_{\omega}: \Pi(X, \perp) \rightarrow[0,1]$, where for each $D^{\perp \perp}$ in $\Pi(X, \perp), \alpha_{\omega}\left(D^{\perp \perp}\right)=\Sigma_{d \in D} \omega(d)$. This mapping is well defined, and it satisfies usual requirements both of a quantum mechanical state and of a probability measure. In quantum mechanics, one often requires that the set of states be "full" in the sense that the order relation on the set of propositions can be recaptured from a knowledge of the behavior of the states alone. In the FoulisRandall approach, a full set of regular states is a set $\Delta$ of regular states with the property that if $C, D \in O(X, \perp)$ and $\alpha\left(C^{\perp \perp}\right) \leqslant \alpha\left(D^{\perp \Perp}\right)$ for all $\alpha$ in $\Delta$, then $C^{\perp \perp} \subset$ $D^{\perp 1}$. A set $\Omega$ of weights is said to be full provided the set $\left\{\alpha_{\omega} \mid \omega \in \Omega\right\}$ is a full set of regular states. Of particular significance are those sample spaces which admit full sets of dispersion free weight functions (weight functions assuming only the values 0 and 1 ). In this case, other weights may arise as convex linear combinations of the dispersion free weights, indicating a possible way in which "hidden variables" may be present. We remark that a dispersion free state in quantum mechanics is usually interpreted to be one in which the physical system is completely and precisely determined.

It can be shown (see [3]) that any sample space which admits a full set of weight functions (and, in particular, a full set of dispersion free weights) must be a Dacey space. The converse does not hold: in fact, there exist Dacey spaces admitting no weight functions whatsoever (see [5]). However, it happens that if every maximal orthogonal set in $(X, 1)$ has a nonempty intersection with every maximal scattered set, then $(X, 1)$ admits a full set of dispersion free weight functions. Observing that a dispersion free weight function is precisely the characteristic function of some maximal scattered set, one can phrase this condition equivalently by requiring that the characteristic function of every set $S$ in $S(X, 1)$ is a (dispersion free) weight function. Note that the characteristic function for $S=$ $\{a, d, f\}$ in Figure 1(c) is not a weight function: its sum is 0 on the operation $E=$ $\{b, c, e\}$ because $E \cap S=\varnothing$. A sample space $(X, \perp)$ with the property that whenever $E \in E(X, \perp)$ and $S \in S(X, \perp)$, then $E \cap S \neq \varnothing$, is called a finitary space. If $(X, \perp)$ is a finitary HD space, it is usually designated an FHD space. In Lemma 4, we will show that every finite HD space is an FHD space, whence the name "finitary."

FHD spaces are particularly important for three basic reasons. As HD spaces, they can reasonably be assumed appropriate representations of quantum mechanical systems. Furthermore, as finitary spaces they generalize those sample spaces depicting physical situations which are, in a sense, physically realizable. In addition, 
such sample spaces always admit full sets of dispersion free weight functions, and thus, hidden variables.

In this paper, we will describe a method by which any HD space may be imbedded, in a canonical and natural way, in an FHD sample space.

II. Idealized points in an HD space. Let us begin with an example. Suppose $n$ is a positive integer. Let the directions for an operation $E_{n}$ read "flip a coin repeatedly until it comes up 'heads' or until the coin has been thrown $n$ times, whichever occurs first. If 'heads' appears on the $r$ th throw, $r \leqslant n$, record the outcome $t^{r-1} h$. If 'heads' has not appeared after $n$ throws, record $t^{n}$." Thus

$$
E_{n}=\left\{t^{r-1} h \mid r=1,2,3, \cdots, n\right\} \cup\left\{t^{n}\right\} \quad\left(t^{0} h=h\right) .
$$

Let $X=\bigcup_{n=1}^{\infty} E_{n}=\left\{t^{r-1} h \mid r=1,2,3, \cdots\right\} \cup\left\{t^{n} \mid n=1,2,3, \cdots\right\}$. We define elements $x$ and $y$ in $X$ to be orthogonal if and only if $x \neq y$ and there exists an integer such that $x, y \in E_{n}$. Assuming that there are only two possible outcomes, "heads" and "tails," for each throw of the coin, the maximal orthogonal subsets of $X$ are precisely the $E_{n}$ 's for $n=1,2, \cdots$, together with the set $E=$ $\left\{t^{r-1} h \mid r=1,2,3, \cdots \cdot\right.$. The maximal scattered sets are: $S_{n}=\left\{t^{r} \mid r=1,2,3\right.$, $\cdots, n-1\} \cup\left\{t^{n-1} h\right\}$ for $n=1,2,3, \cdots$, and the set $S=\left\{t^{r} \mid r=1,2,3\right.$, $\cdots$. This example is easily visualized as a subset of the "free orthogonality monoid" over a two element "base space" $\{h, t\}$ as depicted in [8, pp. 365-366], [9], [11], and [12]. The sample space of this example, while an HD space, is not an FHD space, since $E \cap S=\varnothing$. That these two sets have an empty intersection in $X$ is due to the absence in $X$ of the "in-principle" outcome $t^{\infty}$, representing the theoretical possibility that the coin may be tossed repeatedly, yielding an unbounded succession of "tails."

Let us make the convention that henceforth $(X, 1)$ will represent an arbitrary $\mathrm{HD}$ space. Our example suggests that missing "in-principle" outcomes in an $\mathrm{HD}$ space are characterized by the presence of a maximal orthogonal set and a maximal scattered set which have empty intersection. If $(E, S) \in E(X, 1) \times S(X, 1)$ where $E \cap S=\varnothing$, we agree to represent the missing outcome these sets conceal by the ordered pair $(E, S)$, called an idealized point. Let $X^{\prime}$ denote the collection of all idealized points associated with $X$ (this definition will be slightly modified presently), and let $\bar{X}=X \cup X^{\prime}$. Equipped with a natural extension of the orthogonality on $X, \bar{X}$ will be the FHD space in which we will imbed $X$.

Before extending the notion of orthogonality from $X$ to $\bar{X}$, however, we first observe that the scattered set $S$ of an idealized point $(E, S)$ may be ordered in a very natural way. In the coin-tossing example presented above, for $t^{j}$ and $t^{k}$ in $S$, we might define $t^{j}$ to be less than $t^{k}$ if $j>k$, or, very informally, if $t^{j}$ takes longer to obtain as an outcome than does $t^{k}$. This is the case if and only if there 
is some outcome in $E, t^{j-1} h$ for instance, which operationally rejects $t^{j}$, but does not reject $t^{k}$. We adopt this as our definition.

1. Definition. Let $(E, S)$ be in $X^{\prime}$, and let $s_{1}, s_{2} \in S$. Define $s_{1}<s_{2}(E, S)$ if and only if there is some element $e$ in $E$ such that $e \perp s_{1}$ and $e \# s_{2}$.

Such a situation is depicted in Figure 3(a) where the role of the element $e$ in the definition is taken by $e_{1}$. While the relation defined above is clearly dependent on the idealized point chosen, where no possibility of confusion exists, $s_{1}<$ $s_{2}(E, S)$ will be shortened to $s_{1}<s_{2}$. We define $s_{1} \leqslant s_{2}$ if $s_{1}<s_{2}$ or $s_{1}=s_{2}$.

2. Lemma. For any $(E, S)$ in $X^{\prime}$, the relation $\leqslant$ is an order relation on $S$.

Proof. Reflexivity is clear. We will demonstrate the antisymmetry of the relation, noting that the proof of transitivity is similar. Let $s_{1} \leqslant s_{2}$ and $s_{2} \leqslant s_{1}$, and suppose that $s_{1} \neq s_{2}$. Then since $s_{1}<s_{2}$, there exists $e_{1}$ in $E$ as in Figure 3(a), while $s_{2}<s_{1}$ implies the existence of $e_{2}$ in $E$ situated as in Figure 3(b). Noting that $e_{1}$ and $e_{2}$ are distinct elements of $E$ and therefore orthogonal, we combine these to obtain Figure 3(c), a hook and a contradiction.

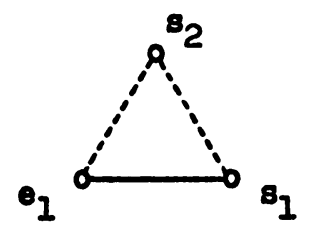

(a)

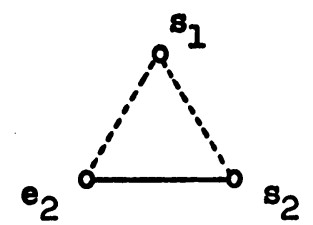

(b)

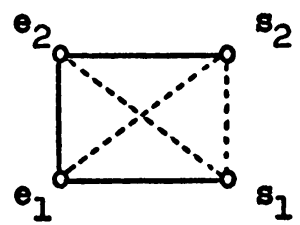

(c)

\section{FiguRe 3}

Still considering the idealized point $(E, S)$, we remark that a totally ordered set may be obtained from the order relation on $S$ as follows. For $s_{1}, s_{2}$ in $S$, define $s_{1} \sim s_{2}(E, S)$ whenever $s_{1}^{\perp} \cap E=s_{2}^{\perp} \cap E$. Once again, $(E, S)$ will be suppressed in the notation where possible. Then, writing $s_{1} \lesssim s_{2}$ if $s_{1}<s_{2}$ or $s_{1} \sim s_{2}$, the relation $\lesssim$ clearly renders $S$ modulo this equivalence relation a totally ordered set. This relation " " will be instrumental in $\S \mathrm{V}$.

We now define an order relation on the first component of $(E, S)$ in a similar fashion.

3. Definition. Let $(E, S) \in X^{\prime}$ and $e_{1}, e_{2} \in E$. Define $e_{1}<e_{2}(E, S)$ if and only if there exists some $s$ in $S$ such that $s \# e_{1}$ and $s \perp e_{2}$.

The situation is completely dual to that of the order relation on $S: \leqslant$ is an order relation on $E$; $\lesssim$ (where $e_{1} \sim e_{2}$ if and only if $e_{1}^{\#} \cap S=e_{2}^{\#} \cap S$ ) is a total order relation on the equivalence classes of the relation " $\sim$ ".

The following lemma provides a proof of the fact that every finite HD space is finitary. It implies that the existence of an idealized point entails the existence 
of infinitely many distinct elements in each component of the idealized point.

4. Lemma. Let $(E, S) \in X^{\prime}$. Then:

(i) if $e_{1} \in E$, there exists $e$ in $E$ such that $e<e_{1}$;

(ii) if $s_{1} \in S$, there exists $s$ in $S$ such that $s<s_{1}$.

Proof. We show (i); (ii) is similar. Let $e_{1}$ be in $E$. Then there is an $s^{\prime}$ in $S$ such that $s^{\prime} \perp e_{1}$; for if not, since $e_{1} \notin S$, we would have $e_{1} \in S^{\#}$. But this contradicts the fact that $S^{\#}=\varnothing, S$ being a maximal scattered set. Now since $s^{\prime} \in S$, we know that $s^{\prime} \notin E$, so that there must exist some element $e$ in $E$ such that $e \# s^{\prime}$. Hence, by the element $s^{\prime}$, we have $e<e_{1}$.

III. Orthogonality in $\bar{X}$. Surely we want the orthogonality relation which we eventually define on $\bar{X}$ to be an extension of the orthogonality in $X$, so that elements of $X$ are pairwise orthogonal if and only if they are orthogonal in $\bar{X}$. Recalling that the components of an idealized point $(E, S)$ can be ordered, it is natural to define an element $x$ in $X$ to be orthogonal to the "in-principle" outcome $(E, S)$ if, from some point on, $x$ is orthogonal to all $e$ in $E$, and to all $s$ in $S$. Thus, for instance, in the coin-throwing example of the preceding section, $t^{n} h$ would be orthogonal to $t^{\infty}$ (which corresponds to $(E, S)$ ) for any integer $n$.

5. Lemma. Let $x \in X,(E, S) \in X^{\prime}$. Then the following two statements are equivalent:

(i) There exist $e$ in $E$ and $s$ in $S$ such that $e \# s$ and $x \in e^{\perp} \cap s^{\perp}$ (see Figure 4(a)).

(ii) There exist $e$ in $E$ and $s$ in $S$ such that $x \perp e_{1}$ for all $e_{1} \leqslant e$, and $x \perp s_{1}$ for all $s_{1} \leqslant s$.

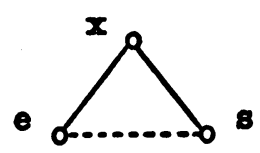

(a)

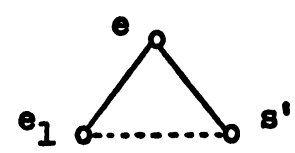

(b)

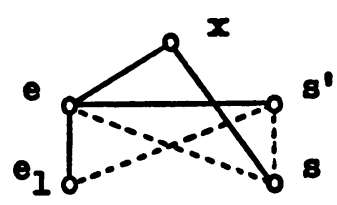

(c)

FIGURE 4

Proof. Assume (i), and let $e_{1}<e$. (If $e_{1}=e$, then $x \perp e$ is trivial.) Then there exists $s^{\prime} \in S$ as in Figure 4(b). Combining diagrams 4(a) and 4(b) we have the diagram in Figure 4(c). But to avoid the potential hook $\left(s, e_{1}, e, s^{\prime}\right)$, we must have that $e_{1} \# s$. It then follows that $x \perp e_{1}$, for if not, $\left(e_{1}, e, x, s\right)$ is a hook. Thus $x \perp e_{1}$ for all $e_{1} \leqslant e$. A similar argument yields that $x \perp s_{1}$ for all $s_{1} \leqslant s$.

Conversely, we assume that (ii) holds. Note that if $e \# s$, then (i) is immediate. So suppose that $e \perp s$. Then since $s \notin E$, there is some $e_{1}$ in $E$ with $e_{1} \# s$. 
Thus by definition $e_{1}<e$, whence $x \perp e_{1}$ by (ii). But this yields the hoped-for conclusion $e_{1} \# s, x \in e_{1}^{\perp} \cap s^{\perp}$.

The following corollary is a consequence of the proof of the above lemma.

6. Corollary. If $x \in X,(E, S) \in X^{\prime}$, and $e \in E, s \in S$, such that $e$ \# $s$ and $x \in e^{\perp} \cap s^{\perp}$, then $x \in e_{1}^{\perp} \cap s_{1}^{\perp}$ for all $e_{1} \leqslant e$ and for all $s_{1} \leqslant s$.

In view of Lemma 5 and our previous considerations, we define $x \perp(E, S)$ whenever (i) or (ii) of Lemma 5 holds. To ensure symmetry, define $(E, S) \perp x$ if and only if $x \perp(E, S)$. Henceforth, for $x \in X$, the notation $x^{\perp}$ will be extended to mean all those elements of $\bar{X}$ which are orthogonal to $x$.

Since every element of $E$ lies strictly above another element of $E$ in the order relation, the definition of that order relation on $E$ ensures that $e \perp(E, S)$ for all $e$ in $E$. On the other hand, given any $s$ in $S$, one cannot find another element of $S$ orthogonal to $s$, so that $s \notin(E, S)^{\perp}$.

The reader is encouraged to follow subsequent proofs with orthogonality diagrams to add to their clarity. We extend the preceding lemma as follows.

7. Lemma. Let $(E, S),(F, T)$ be in $X^{\prime}$. Then the following two statements are equivalent:

(i) There exist $e \in E, s \in S$ such that $e \# s$ and $(F, T) \in e^{\perp} \cap s^{\perp}$.

(ii) There exist $e \in E, s \in S$ such that $(F, T) \perp e_{1}$ for all $e_{1} \leqslant e$, and $(F, T)$ $\perp s_{1}$ for all $s_{1} \leqslant s$.

Proof. Assume first that (i) holds for $e \in E, s \in S$. Then since $e \perp(F, T)$ and $s \perp(F, T)$, there exist $f_{1}, f_{2}$ in $F$ and $t_{1}, t_{2}$ in $T$ such that $f_{i} \# t_{i}$ for $i=1,2$, and $e \in f_{1}^{\perp} \cap t_{1}^{\perp}, s \in f_{2}^{\perp} \cap t_{2}^{\perp}$. Now let $t \in T$ such that $t \leqslant t_{i}(F, T)$ for $i=1,2$. Then there exists $f_{3}$ in $F$ for which $f_{3} \# t$. Choose $f \in F$ where $f \leqslant f_{i}(F, T)$ for $i=1,2,3$. The preceding corollary gives us $e \perp t, s \perp t$, and $e \perp f, s \perp f$. We also claim that $f \# t$, for suppose not: then $f_{3} \# t$, and $f \neq f_{3}$ so that $f \in f_{3}^{\perp} \cap t^{\perp}$, yielding $f_{3}<f(F, T)$, a contradiction to the choice of $f$. Thus we have Figure 5 .

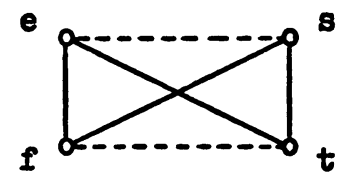

FIGURE 5

Now another application of the corollary to the idealized point $(E, S)$ gives that $e_{1} \in f^{\perp} \cap t^{\perp}$ for all $e_{1} \leqslant e$. Thus for any $e_{1} \leqslant e$, we have that $e_{1} \perp(F, T)$. Similarly, $s_{1} \perp(F, T)$ for any $s_{1} \leqslant s$, and we have demonstrated that (i) implies (ii). For the argument in the other direction, we need only follow the proof of the corresponding implication in Lemma 5 , replacing $x$ by $(F, T)$. 
We define $(E, S) \perp(F, T)$, or $(F, T) \perp(E, S)$ whenever either of the conditions in the statement of Lemma 7 holds. Now the notation $(E, S)^{\perp}$, and all other orthogonality space notations, will have a well-defined meaning in the orthogonality space $(\bar{X}, \perp)$.

To summarize, we have taken an HD space $(X, \perp)$, added certain "idealized" outcomes, and extended the definition of orthogonality to obtain a new orthogonality space $(\bar{X}, \perp)$. Now considering the complement of $(X, \perp)$, namely $(X, \#)$, as an $\mathrm{HD}$ space, where orthogonality is "\#", one can expand to $(\bar{X}, \#)$ by the same process. The question now arises as to whether or not $(\bar{X}, \#)$ obtained in this fashion is the complement of $(\bar{X}, \perp)$, where an idealized point $(E, S)$ in $(\bar{X}, \perp)$ is identified with $(S, E)$ in $(\bar{X}, \#)$. To show that this is the case, we must demonstrate that whenever $x$ and $y$ are distinct elements of $\bar{X}$, then $x \perp y$ fails in $(\bar{X}, \perp)$ precisely when $x \# y$ holds in $(\bar{X}, \#)$. This is clear if $x, y \in X$. Thus we need consider only two cases: (i) $x \in X, y \in X^{\prime}$; (ii) $x, y \in X^{\prime}$. The following theorem yields case (i).

8. Theorem. Let $x \in X,(E, S) \in X^{\prime}$. Then $x \perp(E, S)$ in $(\bar{X}, \perp)$ fails if and only if $x \#(S, E)$ in $(\bar{X}, \#)$.

Proof. Suppose $x \perp(E, S)$ in $(\bar{X}, \perp)$ and $x \#(S, E)$ in $(\bar{X}, \#)$ simultaneously fail. If $x$ were in $E, x \perp(E, S)$ in $(\bar{X}, \perp)$ would hold; therefore $x \notin E$. Similarly, $x \notin S$. Thus we can find $e$ in $E$ such that $e \# x$, and $s$ in $S$ such that $s \perp x$. Suppose $e \# s$. There exists $s_{1}$ in $S$ with $s_{1} \perp e$. Since $\left(e, s_{1}, x, s\right)$ is not a hook, necessarily $x \# s_{1}$. Now $x \in e^{\#} \cap s_{1}^{\#}$ and $e \perp s_{1}$ together yield $x \#(S, E)$, a contradiction. On the other hand, if $e \perp s$, we can find $e_{1}$ in $E$ with $e_{1} \# s$. Now $\left(x, s, e, e_{1}\right)$ may not be a hook, so that $x \perp e_{1}$. But then since $x \in e_{1}^{\perp} \cap s^{\perp}$ and $e_{1} \# s$, we have that $x \perp(E, S)$, another contradiction.

Conversely, suppose that $x \#(S, E)$ in $(\bar{X}, \#)$ and $x \perp(E, S)$ in $(\bar{X}, \perp)$. By definition, then, we can find $e, e_{1}$ in $E, s, s_{1}$ in $S$ as illustrated in Figure 6(a) and (b). Combining these, we have Figure 6(c). If $s \perp e_{1}$, then

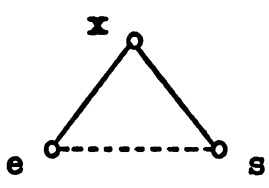

(a)

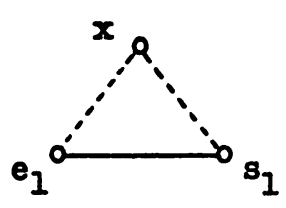

(b)

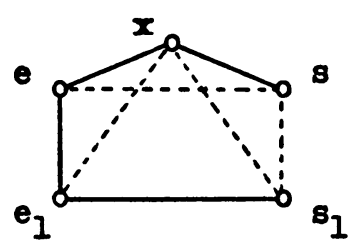

(c)

\section{FIGURE 6}

$\left(x, s, e_{1}, s_{1}\right)$ is a hook, a contradiction. Thus $e_{1} \# s$, making $\left(s, x, e, e_{1}\right)$ a hook, and a final contradiction. 
We now ask if it is necessarily true that two idealized points $(E, S)$ and $(F, T)$ are either orthogonal in $(\bar{X}, 1)$ or orthogonal $(\#)$ in $(\bar{X}, \#)$. Unfortunately, it is possible that $(E, S) \perp(F, T)$ in $(\bar{X}, \perp)$ and $(S, E) \#(T, F)$ in $(\bar{X}, \#)$ may fail simultaneously. When this occurs, however, the ideal points are essentially the same, for one can show that $(E, S)^{\perp} \cap \bar{X}=(F, T)^{\perp} \cap \bar{X}$. We define an obvious equivalence relation on the idealized points which eliminates the difficulty: say that $(E, S) \sim(F, T)$ if and only if $(E, S) \perp(F, T)$ in $(\bar{X}, \perp)$ and $(S, E) \#(T, F)$ in $(\bar{X}, \#)$ simultaneously fail. At this point we redefine $X^{\prime}$ to be an exhaustive set of mutually nonequivalent idealized points; as before, $\bar{X}=X \cup X^{\prime}$. With this modification, it is easy to see that the complement of $(\bar{X}, \perp)$ is precisely that sample space yielded by complementing $(X, \perp)$ to obtain $(X, \#)$, and then adjoining $X^{\prime}$ and extending the orthogonality \#. More graphically, if " $k$ " denotes the operation of taking the complement, and if "-" denotes the operation of expanding, we have that the following diagram is commutative.

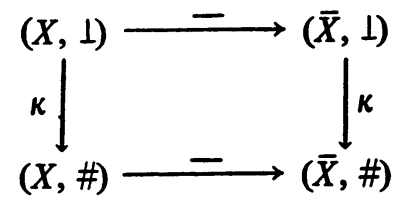

IV. $(\bar{X}, \perp)$ as an HD space. In this section we will show that if $(X, 1)$ is an $\mathrm{HD}$ space, then so is the extended space $(\bar{X}, 1)$. We shall, of course, proceed by showing that $\bar{X}$ is "hook deficient." Since $(X, \perp)$ is HD, then $(\bar{X}, \perp)$ can contain no hooks consisting solely of elements of $X$. Thus it suffices to show that $(\bar{X}, \perp)$ contains no hooks comprising one or more elements of $X^{\prime}$. The following lemma asserts that given any idealized point and a finite subset of $\bar{X}$, that idealized point can be represented by some element of $X$ whose orthogonality relationships to that finite subset are the same as those of the idealized point. And so if $\bar{X}$ were to contain a hook, each idealized point in this finite configuration could be replaced, in turn, by an appropriate representative in $X$ which would be related to the other three elements of the hook in the same way as the idealized point. But the result of this process is a hook in $(X, 1)$, a contradiction. Thus the lemma immediately implies the desired result.

9. Lemma. If $(E, S) \in X^{\prime}, A, B \subset \bar{X}$, where $A \cup B$ is finite, and if $(E, S) \in$ $A^{\perp} \cap B^{\#}$, then there is an $e$ in $E$ such that $e \in A^{\perp} \cap B^{\#}$.

Proof. Since $A \cup B$ is finite, $A \cup B=\left\{c_{1}, c_{2}, \cdots, c_{n}\right\}$ for some integer $n$. If $c_{i} \perp(E, S)$, let $e_{i}$ denote an element of $E$ with the property that $c_{i} \perp e$ for all $e \leqslant e_{i}(E, S)$ (recall that such an $e_{i}$ exists by the way we defined $c_{i} \perp(E, S)$ ). If, on the other hand, $c_{i} \#(E, S)$ in $(\bar{X}, \perp)$, then $c_{i} \#(S, E)$ in $(\bar{X}, \#)$. We then can choose some $e_{i}$ in $E$ with the property that $c_{i} \# e$ for all $e \leqslant e_{i}(S, E)$, or 
equivalently, for all $e \leqslant e_{i}(E, S)$. Now choose some $e^{\prime}$ in $E$ with $e^{\prime}<e_{i}(E, S)$

for all $i$. If, for some $i, c_{i} \in A$, we have $c_{i} \perp e_{i}$, whence $c_{i} \perp e^{\prime}$ since $e^{\prime}<e_{i}(E, S)$. On the other hand, suppose $c_{i} \in B$. We have $c_{i} \# e_{i}$, and thus $c_{i} \# e^{\prime}$ because $e^{\prime}<e_{i}(S, E)$. We conclude that $e^{\prime} \in A^{\perp} \cap B^{\#}$.

Our earlier discussion yields:

10. THEOREM. If $(X, 1)$ is an HD space, then $(\bar{X}, 1)$ is an HD space also.

V. $(\bar{X}, \perp)$ as a finitary space. It is clear that the operations in $(X, \perp)$ are not the same as those in $(\bar{X}, 1)$; by adjoining to $X$ its set of idealized points with the extended notion of orthogonality, new operations have been created, and old ones destroyed. In the coin-throwing example of $\S I I$, for instance, $E \cup\{(E, S)\} \in$ $E(\bar{X}, \perp)$, while $S \cup\{(E, S)\} \in S(\bar{X}, \perp)$. It is not difficult to see that this particu$\operatorname{lar}(\bar{X}, \perp)$ is an FHD space; that is, every operation in $(\bar{X}, \perp)$ has a nonempty intersection with every maximal scattered set in $(\bar{X}, 1)$. Our goal in this section is to prove this result for an arbitrary "extended" $\mathrm{HD}$ space, $(\bar{X}, 1)$.

Let us suppose, to the contrary, that $(X, 1)$ is an $\mathrm{HD}$ space but that its associated $(\bar{X}, \perp)$, while an HD space, is not finitary. Then there exist sets $\bar{E}$ in $E(\bar{X}, \perp)$ and $\bar{S}$ in $S(\bar{X}, 1)$ with the property that $\bar{E} \cap \bar{S}=\varnothing$. Note that this makes $(\bar{E}, \bar{S})$ an idealized point for $(\bar{X}, \perp)$. Our contradiction will follow when we find an idealized point, say $(H, V)$, in $X^{\prime}$ such that $(H, V) \in \bar{E} \cap \bar{S}$. We note that $(\bar{E}, \bar{S})$ bears a suspicious resemblance to our hypothetical $(H, V)$, with the exception that $H \cup V \subset X$, while $\bar{E} \cup \bar{S} \subset \bar{X}$. Thus we want, somehow, not only to preserve the orthogonality structure of $\bar{E}$ and $\bar{S}$, but also to express it in terms of appropriate elements of $X$, to obtain a suitable $(H, V) \in \bar{E} \cap \bar{S}$. The following claim is a step in the right direction: it asserts that one can replace any element of $\bar{E}$, in particular an element of $\bar{E} \cap X^{\prime}$, by an element of $X$ which has the same orthogonality relationships to elements of $\bar{S}$ as did the original element of $\bar{E}$.

Claim 1. For each $e$ in $\bar{E}$, there is an $h$ in $X$ such that $h \notin e^{\perp}, h \notin \bar{S}$, and $\bar{S} \cap e^{\perp}=\bar{S} \cap h^{\perp}$.

Proof. Let $e$ be in $\bar{E}$. Then if $e \in X$, let $h=e$, and clearly the condition is satisfied. So suppose that $e=(F, T) \in \bar{E} \cap X^{\prime}$. Since $\bar{E} \cap \bar{S}=\varnothing$, there exists some $s_{1}$ in $\bar{S}$ with $s_{1} \perp e$. Thus $s_{1} \perp(F, T)$, so that there exists an $h$ in $T$ such that $s_{1} \perp h$. Now because $h \in T$, we have $h \#(F, T)$, yielding Figure 7(a).

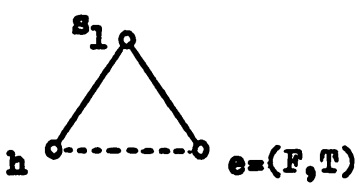

(a)

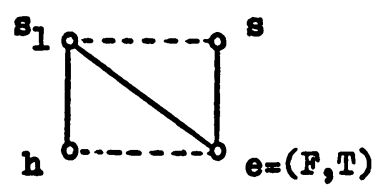

(b)

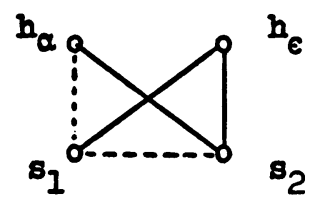

(c)

FIGURE 7 
We use the fact that $\bar{X}$ is hook-deficient to show that $\bar{S} \cap e^{\perp} \subset \bar{S} \cap h^{\perp}$. A complementary argument would show that $\bar{S} \cap e^{\#} \subset \bar{S} \cap h^{\#}$ yielding the equality we seek. Suppose $s \in \bar{S} \cap e^{\perp}$ and $s \neq s_{1}$. (If $s=s_{1}$, the result is clear.) Then Figure 7(b) implies that $s \in h^{\perp}$, and we are done.

Recall that in §III an equivalence relation was mentioned in connection with totally ordering the equivalence classes of $E$ and of $S$, where $(E, S) \in X^{\prime}$. Noting that $(\bar{E}, \bar{S}) \in(\bar{X})^{\prime}$, we can easily extend the notion of equivalence, so that for $e_{1}$, $e_{2}$ in $\bar{E}$ and $s_{1}, s_{2}$ in $\bar{S}$, we define:

(i) $e_{1} \sim e_{2}(\bar{E}, \bar{S})$ if and only if $e_{1}^{\#} \cap \bar{S}=e_{2}^{\#} \cap \bar{S}$;

(ii) $s_{1} \sim s_{2}(\bar{E}, \bar{S})$ if and only if $s_{1}^{\perp} \cap \bar{E}=s_{2}^{\perp} \cap \bar{E}$. Now $\bar{E}$, in particular, is partitioned by the resulting equivalence classes. In each such equivalence class, choose and fix some $e_{\alpha}$, and let $\hat{E}=\left\{e_{\alpha}\right\}$, the set of all these representatives. By the preceding claim, for each $\alpha$, we may choose and fix some $h_{\alpha}$ in $X \backslash e_{\alpha}^{\perp}, h_{\alpha} \notin \bar{S}$, such that $\bar{S} \cap h_{\alpha}^{\perp}=\bar{S} \cap e_{\alpha}^{\perp}$. Let $\hat{H}=\left\{h_{\alpha}\right\}$. We now show that the elements of $X$ so chosen are mutually orthogonal.

Claim 2. $\hat{H} \in O(X, 1)$.

Proof. Let $h_{\alpha}$ and $h_{\epsilon}$ be distinct elements of $\hat{H}$. Then $h_{\alpha}$ and $h_{\epsilon}$ correspond to $e_{\alpha}$ and $e_{\epsilon}$, respectively, in $\hat{E}$, whence $e_{\alpha}$ and $e_{\epsilon}$ must have the property that $e_{\alpha} \nsucc e_{\epsilon}(\bar{E}, \bar{S})$. Then, without loss of generality, we may assume that $e_{\alpha}^{\#} \cap \bar{S} \not \subset e_{\epsilon}^{\#} \cap \bar{S}$. Thus, there exists an element $s_{1}$ in $\bar{S}$ where $s_{1} \in e_{\alpha}^{\#} \backslash e_{\epsilon}^{\#}$. Since $\bar{E} \cap \bar{S}=\varnothing, s_{1} \notin \bar{E}$, so that $s_{1} \perp e_{\epsilon}$. We can also find $s_{2}$ in $\bar{S}$ such that $s_{2} \perp e_{\alpha}$, else $e_{\alpha} \in \bar{S}^{\#}=\varnothing$. But $\left(s_{2}, e_{\alpha}, e_{\epsilon}, s_{1}\right)$ may not be a hook, so that $s_{2} \perp e_{\epsilon}$. And now, our choice of $h_{\alpha}$ and $h_{\epsilon}$ demands that the configuration of Figure 7(c) must also hold. But then $\left(s_{1}, h_{\epsilon}, s_{2}, h_{\alpha}\right)$ is not a hook, so that necessarily $h_{\alpha} \perp h_{\epsilon}$, as required.

Now that we know that $\hat{H}$ is an orthogonal set in $X$, we can expand it to a set $H$ in $E(X, \perp)$, and then expand $H$ to a set $\bar{H}$ in $E(\bar{X}, \perp)$.

\section{Claim 3. $\bar{H} \cap \bar{S}=\varnothing$.}

Proof. Suppose that the intersection is not empty, and let $c \in \bar{H} \cap \bar{S}$. Then, since $c \in \bar{S}$, there is an $e$ in $\bar{E}$ such that $e \# c$. We have previously chosen $e_{\alpha}$ in $\bar{E}$ with $e_{\alpha} \sim e(\bar{E}, \bar{S})$. Then, because $c \in \bar{S}$ and $c \# e$, we have $c \# e_{\alpha}$, and thus $c \# h_{\alpha}$. But this is a contradiction, since $c$ and $h_{\alpha}$ are elements of $\bar{H}$, an orthogonal set. Hence $\bar{H} \cap \bar{S}=\varnothing$.

Thus $\bar{H} \in E(\bar{X}, \perp), \bar{S} \in S(\bar{X}, \perp)$, and $\bar{H} \cap \bar{S}=\varnothing$, so that $(\bar{H}, \bar{S}) \in(\bar{X})^{\prime}$. Note that $(\bar{H}, \bar{S})$ closely resembles $(\bar{E}, \bar{S})$, but that it has the additional property that $H=\bar{H} \cap X \in E(X, 1)$. If we could, in an analogous manner, replace $\bar{S}$ by some $\bar{V}$ in $S(\bar{X}, \perp)$ where $V=\bar{V} \cap X \in S(X, 1)$, then $(H, V)$ would be in $X^{\prime}$.

So we switch our vantage point and evaluate the situation in the comple- 
mentary space $(\bar{X}, \#)$. Observe that $\bar{S} \in E(\bar{X}, \#), \bar{H} \in S(\bar{X}, \#)$, and that $(\bar{X}, \#)$, being the complement of an HD space, is again HD. Thus we can proceed in complete analogy to our previous method of attack, doing to $(\bar{S}, \bar{H})$ in $(\bar{X}, \#)$ what we did to $(\bar{E}, \bar{S})$ in $(\bar{X}, \perp)$. Introducing the appropriate notation, we write down the corresponding claims, emphasizing that we are simply translating our first three claims so that they apply to $(\bar{S}, \bar{H})$ in the complementary space, $(\bar{X}, \#)$.

Claim $1^{\prime}$. For each $s$ in $\bar{S}$, there is $a v$ in $X$ such that $v \notin s^{\#}, v \notin \bar{H}$, and $\bar{H} \cap s^{\#}=\bar{H} \cap v^{\#}$.

Note that, for $s_{1}$ and $s_{2}$ in $\bar{S}, s_{1} \sim s_{2}(\bar{S}, \bar{H})$ in $(\bar{X}, \#)$ if and only if $s_{1}^{\perp} \cap \bar{H}$ $=s_{2}^{1} \cap \bar{H}$, which is equivalent to saying that $s_{1} \sim s_{2}(\bar{H}, \bar{S})$ in $(\bar{X}, 1)$. Now in each equivalence class of elements of $\bar{S}$ formed by this relation, we choose and fix some $s_{\alpha}$. Let $\hat{S}=\left\{s_{\alpha}\right\}$. Then, for each $s_{\alpha}$, Claim (1') allows us to pick and fix some $v_{\alpha}$ in $X \backslash s_{\alpha}^{\#}, v_{\alpha} \notin \bar{H}$, such that $\bar{H} \cap s_{\alpha}^{\#}=\bar{H} \cap v_{\alpha}^{\#}$.

Claim $2^{\prime} . \hat{V}=\left\{v_{\alpha}\right\} \in O(X, \#)$.

Expand $\hat{V}$ to $V$ in $E(X, \#)$, and $V$ to $\bar{V}$ in $E(\bar{X}, \#)$.

Claim 3'. $\bar{V} \cap \bar{H}=\varnothing$.

Since $V \in E(X, \#)$ and $\bar{V} \in E(\bar{X}, \#)$, we have that $V \in S(X, 1)$ and $\bar{V} \in$ $S(\bar{X}, 1)$. We have thus constructed our element $(\bar{H}, \bar{V})$ of $(\bar{X})^{\prime}$. But what is more appealing, $(H, V)$ is an element of $X^{\prime}$, since $H \cap V \subset \bar{H} \cap \bar{V}=\varnothing$.

Suppose that $(H, V) \notin \bar{S}$. Then there must exist some $s$ in $\bar{S}$ such that $s \perp$ $(H, V)$, or else $(H, V)$ would be in $\bar{S}^{\#}$, which is empty. Since $s \perp(H, V)$, there exist $h$ in $H$ and $v$ in $V$ such that $h \# v$ while $s \in h^{\perp} \cap v^{\perp}$. Recalling that $\bar{H} \cap$ $\bar{S}=\varnothing$, we note that there is some $h^{\prime}$ in $\bar{H}$ with $h^{\prime} \# s$. Prior to Claim $2^{\prime}$, we chose an $s_{\alpha}$ in $\hat{S}$ where $s_{\alpha} \sim s(\bar{H}, \bar{S})$. Then $s_{\alpha}^{\perp} \cap \bar{H}=s^{1} \cap \bar{H}$, and so since $h^{\prime} \# s$ we must have that $h^{\prime} \# s_{\alpha}$. We also chose an element $v_{\alpha}$ in $\hat{V}$ which "represents" $s_{\alpha}$ in terms of its relationship to elements of $\bar{H}$. Thus $v_{\alpha} \# h^{\prime}$. In addition $h \perp s$ implies that $h \perp s_{\alpha}$, yielding the fact that $h \perp v_{\alpha}$. Note that $h$ and $h^{\prime}$ are distinct because of $s$, and hence they are orthogonal $\left(h \perp h^{\prime}\right) ; v$ and $v_{\alpha}$ are distinct due to $h$ and therefore sharp. Thus we have Figure 8.

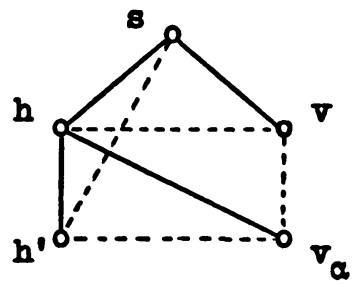

FIGURE 8 
Now to avoid the potential hook $\left(h^{\prime}, h, s, v\right), h^{\prime} \perp v$. But then $\left(v, h^{\prime}, h, v_{\alpha}\right)$ is a hook, giving us a contradiction. So we conclude that $(H, V) \in \bar{S}$, which is half the battle!

Now let us suppose that $(H, V) \notin \bar{E}$. Then there exists some $e$ in $\bar{E}$ such that $e \#(H, V)$. In the lines following the proof of Claim 1, we chose an $e_{\alpha}$ in $E$ such that $e_{\alpha} \sim e(\bar{E}, \bar{S})$, and we chose a corresponding $h_{\alpha}$ in $\hat{H}$. Then $e_{\alpha}^{\perp} \cap \bar{S}=e^{\perp} \cap \bar{S}$, and since $(H, V) \in \bar{S} \backslash e^{\perp}$, we have that $(H, V) \notin e_{\alpha}^{\perp}$, so that $(H, V) \# e_{\alpha}$. Thus $(H, V) \# h_{\alpha}$. But $h_{\alpha} \in \hat{H} \subset H$, so that $h_{\alpha} \perp(H, V)$, a contradiction. Therefore $(H, V) \in \bar{E}$, and we have found an element of $X^{\prime}$ in $\bar{E} \cap \bar{S}$ where this intersection was initially assumed empty. This contradiction gives us our theorem:

11. TheOREM. If $(X, \perp)$ is an HD space, then $(\bar{X}, \perp)$ is an FHD space.

Thus an arbitrary $\mathrm{HD}$ space can be imbedded in a very natural and canonical way in an FHD space. In the light of what we have shown, the procedure of completing an $\mathrm{HD}$ space $(X, 1)$ to obtain $(\bar{X}, 1)$ is called the finitary completion (or F-completion) of $(X, \perp)$.

This procedure seems to enhance the importance of the HD sample space: not only is the logic of such a sample space a complete orthomodular lattice, but, by merely adjoining to the space its "in-principle" outcomes, we also have a space which enjoys many desirable properties of a finite $\mathrm{HD}$ space. For one thing, the new sample space admits a full set of dispersion free weight functions. Furthermore, since the $F$-completion is an FHD space, its logic is atomic (a result in [4]). In these two important ways, then, the enlarged sample space conforms to Mackey's axioms for quantum mechanics and shares the obvious corresponding characteristics of the dispersion free weight functions and the lattice of propositions in a classical ordinary probability space.

\section{REFERENCES}

1. G. Birkhoff, Lattice theory, 3rd ed., Amer. Math. Soc. Colloq. Publ., vol. 25, Amer. Math. Soc., Providence, R. I., 1967. MR 37 \#2638.

2. J. C. Dacey, Orthomodular spaces and additive measurement, Caribbean J. Sci. Math. 1 (1969), 51-66.

3. D. J. Foulis and C. H. Randall, Operational statistics. I: Basic concepts, J. Mathematical Phys. 13 (1972), 1667-1675.

4. M. A. Gaudard, Maximal refinement ideals and atomicity in certain generalized sample spaces (in preparation).

5. R. J. Greechie, Orthomodular lattices admitting no states, J. Combinatorial Theory Ser. A 10 (1971), 119-132. MR 43 \#120.

6. A. N. Kolmogorov, Grundbegriffe der Wahrscheinlichkeitsrechnung, Springer, Berlin, 1933; English transl., Foundations of the theory of probability, 2nd ed., Chelsea, New York, 1956. MR 11, 374; 18, 155.

7. G. W. Mackey, The mathematical foundations of quantum mechanics: A lecturenote volume. Benjamin, New York, 1963. MR 27 \#5501. 
8. C. H. Randall and D. J. Foulis, An approach to empirical logic, Amer. Math. Monthly 77 (1970), 363-374. MR 41 \#3334.

9. - Lexicographic orthogonality, J. Combinatorial Theory Ser. A 11 (1971), 157-162. MR 43 \#6140.

10. - Operational statistics. II: Manuals of operations and their logics, J. Mathematical Phys. 14 (1973), 1472-1480.

11. R. J. Weaver, Admissible operations on sample spaces over the free orthogonality monoid, Colloq. Math 25 (1972), 319-324.

12. - Closed sets in the free orthogonality monoid, Amer. J. Math. 96 (1974), 593-601.

13. - Refinement conditions on operations in sample spaces, Canad. J. Math. (to appear).

DEPARTMENT OF MATHEMATICS, UNIVERSITY OF MASSACHUSETTS, AMHERST, MASSACHUSETTS, 01002

DEPARTMENT OF MATHEMATICS, MOUNT HOLYOKE COLLEGE, SOUTH HADLEY, MASSACHUSETTS 01075 